This is an electronic reprint of the original article. This reprint may differ from the original in pagination and typographic detail.

Author(s): Koskinen, Pekka

Title: Quantum Simulations of One-Dimensional Nanostructures under Arbitrary Deformations

Year: $\quad 2016$

Version:

Please cite the original version:

Koskinen, P. (2016). Quantum Simulations of One-Dimensional Nanostructures under Arbitrary Deformations. Physical Review Applied, 6(3), Article 034014.

https://doi.org/10.1103/PhysRevApplied.6.034014

All material supplied via JYX is protected by copyright and other intellectual property rights, and duplication or sale of all or part of any of the repository collections is not permitted, except that material may be duplicated by you for your research use or educational purposes in electronic or print form. You must obtain permission for any other use. Electronic or print copies may not be offered, whether for sale or otherwise to anyone who is not an authorised user. 


\title{
Quantum Simulations of One-Dimensional Nanostructures under Arbitrary Deformations
}

\author{
Pekka Koskinen* \\ Department of Physics, NanoScience Center, University of Jyvaskyla, 40014 Jyvaskyla, Finland \\ (Received 20 May 2016; revised manuscript received 30 June 2016; published 26 September 2016) \\ A powerful technique is introduced for simulating mechanical and electromechanical properties of \\ one-dimensional nanostructures under arbitrary combinations of bending, twisting, and stretching. The \\ technique is based on an unconventional control of periodic symmetry which eliminates artifacts due to \\ deformation constraints and quantum finite-size effects and allows transparent electronic-structure analysis. \\ Via density-functional tight-binding implementation, the technique demonstrates its utility by predicting \\ nonlinear electromechanical properties in carbon nanotubes and abrupt behavior in the structural yielding \\ of $\mathrm{Au}_{7}$ and $\mathrm{Mo}_{6} \mathrm{~S}_{6}$ nanowires. The technique drives simulations markedly closer to the realistic modeling of \\ these slender nanostructures under experimental conditions.
}

DOI: 10.1103/PhysRevApplied.6.034014

\section{INTRODUCTION}

A significant part of contemporary nanomaterial research investigates one-dimensional (1D) nanostructures. Research motivations originate from a plethora of applications among medicine [1], nanoelectronics [2-5], nanomechanics [6-8], filters [9], sensors [10,11], material reinforcement [12], and the tailoring of material properties $[13,14]$. Some 1D nanostructures are synthesized bottom up, others are fabricated top down [15-20], and some are simply found directly in nature $[21,22]$. Yet, all of these nanostructures share one common feature: extreme slenderness. Because of large aspect ratios, they are prone to bending, twisting, and stretching, along with their arbitrary combinations. Such deformations are ubiquitous in practice, as proven by numerous experiments [23-27].

However, just as eliminating deformations in experiments is hard, incorporating them into theory is even harder. All deformations are possible in finite structures, but related simulations are plagued by problems. First, most nanostructures have so many atoms that their straightforward simulation is simply out of question. Second, quantum simulations of finite structures are often deteriorated by finite-size artifacts. Third, unless specifically designed to mimic experimental settings, mechanical deformation constraints can sabotage the very phenomena under study. Fourth, electronic-structure analysis of finite constrained structures is often cumbersome. Therefore, 1D nanostructures are best treated by periodic boundary conditions, effectively modeling infinite extensions. Various loading conditions were investigated previously, but arbitrary deformations have always been simulated using finite structures $[28,29]$. Also, although recent methodological advances have enabled simulating periodic structures under pure twisting and pure bending [30-33], experimental deformations are

\footnotetext{
"pekka.koskinen@iki.fi
}

rarely pure [34]. Electronic-structure simulations of 1D nanostructures with realistic, arbitrary deformations have remained elusive.

In this article, therefore, I introduce a technique to model 1D nanostructures with arbitrary deformations. Based on revised periodic boundary conditions, the technique eliminates artifacts related to quantum finite-size effects and mechanical deformation constraints. It also allows easy electronic-structure analysis in studies on electromechanics. I demonstrate the utility of the technique by revealing surprises in the electromechanical properties of carbon nanotubes and by predicting unconventional structural yielding behavior in $\mathrm{Au}_{7}$ and $\mathrm{Mo}_{6} \mathrm{~S}_{6}$ nanowires.

\section{THE TECHNIQUE}

\section{A. 1D periodicity with customized symmetry}

To first introduce notations, consider electrons in a potential $V(\boldsymbol{r})$ invariant under an isometric symmetry operation $\hat{D}(\mathcal{S}) V(\boldsymbol{r})=V\left(\mathcal{S}^{-1} \boldsymbol{r}\right)=V(\boldsymbol{r})$, where $\boldsymbol{r}^{\prime}=\mathcal{S} \boldsymbol{r}$. Since the Hamiltonian operator $\hat{H}=-\hbar^{2} /\left(2 m_{e}\right) \nabla^{2}+$ $V(\boldsymbol{r})$ commutes with the operator $\hat{D}(\mathcal{S})$, the two operators share the same eigenstates. If we further assume a periodic boundary condition after $N$ successive operations of $\hat{D}(\mathcal{S})$ - that is, $\hat{D}\left(\mathcal{S}^{N}\right) \equiv \hat{1}$ - the eigenstates acquire the property $\hat{D}\left(\mathcal{S}^{n}\right) \psi_{a k}(\boldsymbol{r})=\exp (i \kappa n) \psi_{a k}(\boldsymbol{r})$, where $n$ is the number of symmetry operations and $\kappa \in[-\pi, \pi]$ is a good quantum number used to index the eigenstates. Consequently, the wave function $\psi_{a \kappa}(\boldsymbol{r})$ within a minimal unit cell determines the wave function in the entire extended structure. If the symmetry operation $\mathcal{S}$ is the translation $\mathcal{T}$, the above is obviously nothing but Bloch's theorem in one dimension [35]. The theorem, however, applies also for symmetries beyond translation, although, as yet, few implementations exploit this feature [30,36]. The formalism of using symmetry and periodicity in this 
generalized fashion is referred to as revised periodic boundary conditions (RPBCs) [32,33].

Consider, then, a one-dimensional nanostructure and the symmetry operation

$$
\boldsymbol{r}^{\prime}=\mathcal{S}_{t} \boldsymbol{r} \equiv \mathcal{R}\left[\hat{z}\left(\varphi_{s}\right)\right]\left\{\boldsymbol{R}+\mathcal{R}\left[\hat{t}\left(\phi_{s}\right)\right](\boldsymbol{r}-\boldsymbol{R})\right\},
$$

where $\mathcal{R}[\hat{u}(\theta)](\vec{v})$ represents the rotation of a vector $\vec{v}$ an angle $\theta$ with respect to the axis $\hat{u}$, where the angles $\varphi_{s}$ and $\phi_{s}$ are small (Fig. 1). The successive operation of $\mathcal{S}_{t}$ thus stands for a bending operation around the $z$ axis combined to a screw operation around a gradually reorienting chiral axis with a radius of curvature $R=|\boldsymbol{R}|$. Compared to previous usages of symmetry, the operation (1) conceals one fundamentally unconventional feature: position dependence of the operation itself. This dependence makes the operation, in principle, nonisometric. It turns out, however, that if $\varphi_{s}$ or $\phi_{s}$ or both are small, and if atom positions $\boldsymbol{r}_{I}$ in the nanostructure have the symmetry $\boldsymbol{r}_{I}^{\prime}=\mathcal{S}_{t} \boldsymbol{r}_{I}$, the property of isometry, the symmetry of the potential $V\left(\mathcal{S}_{t} \boldsymbol{r}\right) \approx V(\boldsymbol{r})$, and the commutation of operators $\left[\hat{D}\left(\mathcal{S}_{t}\right), \hat{H}\right]=0$ become valid approximations. Because of the commutation of operators, in the spirit of revised periodic boundary conditions, $\psi_{a k}(\boldsymbol{r})$ within a unit cell then suffices to describe the entire extended nanostructure (Fig. 1) [32,33]. Although physically sensible structures require that $\varphi_{s}$ and $\phi_{s}$ be integer fractions of $2 \pi$, in practice their smallness renders such requirements irrelevant. That is, by requiring an interaction range to be small compared to $R$, bending, twisting, and stretching can be regarded as local deformations, as established previously [37-39].

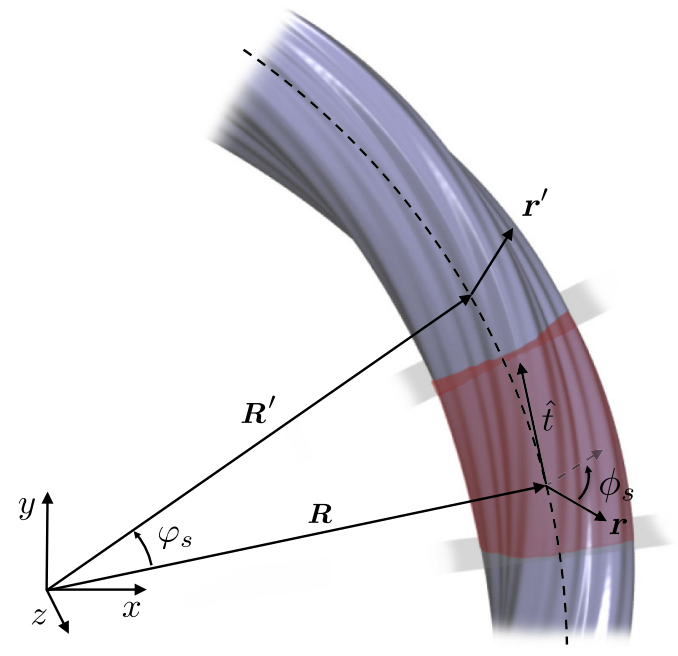

FIG. 1. Deformed 1D nanostructure. Atoms obey the symmetry operation $\boldsymbol{r}^{\prime}=\mathcal{S}_{t} \boldsymbol{r}$ [Eq. (1)]. Arbitrary deformations can be created by simultaneous adjustments of bending (via $\varphi_{s}$ and $R=|\boldsymbol{R}|$ ), twisting (via $\phi_{s}$ ), and stretching (via $R$ and $\varphi_{s}$ ). The chiral-symmetry axis (the dashed curve) lies in the $x-y$ plane. The red shaded area sketches the simulation cell.
Consequently, Eq. (1) becomes the foundation that enables effective modeling of 1D nanostructures with arbitrary deformations. Deformations are controlled via the parameters $R, \varphi_{s}$, and $\phi_{s}$ as follows. First, by considering a structure with diameter $D$, bending can be quantified by the strain $\Theta=D /(2 R)$. In the absence of axial strain, the bending angle is $\varphi_{s}=L_{0} / R$, where $L_{0}$ is the cell length of the undeformed structure, and $\Theta$ equals the maximal tensile and compressive strains along the tangential direction. Second, chiral twisting can be quantified by the sidewall shear $\gamma=(D / 2) \phi_{s} / L_{0}$. Third, axial strain can be quantified simply by $\varepsilon=\left(L-L_{0}\right) / L_{0}$, where $L=R \varphi_{s}$ is the strained axial length. Thus, the three strains $(\Theta, \gamma, \varepsilon)$ fully quantify arbitrary local deformations in 1D nanostructures. Note that deformations are created not by external constraints but by underlying symmetries; all atoms remain fully unconstrained.

\section{B. Density-functional tight-binding implementation}

I implemented the technique using the densityfunctional tight-binding (DFTB) method and the HOTBIT code $[40,41]$. Any classical force field or electronicstructure method would have been equally suitable, but the DFTB formalism allows straightforward implementation and describes the energetics and electronic structures of covalent-and even metallic - systems with reasonable accuracy $[40,42,43]$. The pertinent parametrizations are adopted from Refs. [40,42,43].

According to the RPBC formalism $[32,33]$, the DFTB electron wave functions under the symmetry (1) are described by the revised Bloch basis functions,

$|\kappa, \mu\rangle \equiv \varphi_{\mu}(\kappa, \boldsymbol{r})=\frac{1}{\sqrt{N}} \sum_{n} \exp (-i \kappa n) \hat{D}\left(\mathcal{S}_{t}^{n}\right) \varphi_{\mu}(\boldsymbol{r})$,

where $\varphi_{\mu}(\boldsymbol{r})$ represents a minimal set of local orbitals and $\sum_{n} 1=N$ is the number of unit cells. On this basis, the Hamiltonian is diagonal in $\kappa$,

$$
\left\langle\kappa, \mu|\hat{H}| \kappa^{\prime}, \nu\right\rangle=\delta\left(\kappa-\kappa^{\prime}\right) \sum_{n} \exp (-i \kappa n) H_{\mu \nu}(n),
$$

where the Hamiltonian matrix elements are

$$
H_{\mu \nu}(n)=\int \varphi_{\mu}^{*}(\boldsymbol{r}) \hat{H}\left[\hat{D}\left(\mathcal{S}_{t}^{n}\right) \varphi_{\nu}(\boldsymbol{r})\right] d^{3} r
$$

Together with analogous equations for overlap matrix elements, the DFTB total-energy expression is as usual [40,41], forces are calculated as parametric derivatives of the total energy, and structural relaxation and molecular dynamics are performed in the usual fashion [41]. The positions of atoms' periodic images are mapped exactly via Eq. (1), but, for simplicity, the orbital rotations $\hat{D}\left(\mathcal{S}^{n}\right) \varphi_{\nu}(\boldsymbol{r})$ 
in Eq. (4) are done using the averaged tangential vector of $\hat{t}=-\sin \left(\varphi_{s} / 2\right) \hat{x}+\cos \left(\varphi_{s} / 2\right) \hat{y}$.

Finally, although the concept of a unit cell is familiar, conceptually intuitive, and visually helpful, here such a concept is, in principle, unnecessary. Unit cell is even sketched in Fig. 1, but in practical implementation it is nowhere to be found, because DFTB only requires atoms' relative positions, which are fully determined by Eq. (1). Atoms do not need to remain inside a certain spatial region.

\section{Deformation simulations}

Deformation simulations began with initial guesses for the positions of each atom $I$ that are determined from

$$
\boldsymbol{r}_{I}=\mathcal{R}\left[\hat{z}\left(\chi \varphi_{s}\right)\right]\left\{R \hat{x}+\mathcal{R}\left[\hat{y}\left(\chi \phi_{s}\right)\right]\left(\tilde{x}_{I} \hat{x}+\tilde{z}_{I} \hat{k}\right)\right\},
$$

where $\tilde{\boldsymbol{r}}=\tilde{x}_{I} \hat{x}+\tilde{y}_{I} \hat{y}+\tilde{z}_{I} \hat{k}$ represents atom positions in an undeformed 1D nanostructure centered around the $y$ axis. The length of the structure is $L$, which means that the variable $\chi=\tilde{y}_{I} / L$ ranges from $\chi=0$ to $\chi=1$. Simulations then continue either by structural relaxation, using the fast inertial relaxation engine optimizer [44], or by molecular dynamics simulations using a Langevin thermostat with a 10-K temperature and a 0.5-ps damping time.

Two notes are worth mentioning here. First, if $\phi_{s}=0$, then the symmetry operation becomes

$$
\boldsymbol{r}^{\prime}=\mathcal{S}_{t} \boldsymbol{r} \equiv \mathcal{R}\left[\hat{z}\left(\varphi_{s}\right)\right](\boldsymbol{r}),
$$

which makes $\mathcal{S}_{t}$ independent of $R$. Therefore, in the general case, the radius of curvature for an untwisted, relaxed structure could not be controlled by $\mathcal{S}_{t}$; for a structure with the symmetry $C_{n}$, control can be regained by setting $\phi_{s}=2 \pi / n$, thus mimicking the untwisted structure of a chiral-symmetry operation. Second, the eigenproblem faces occasional technical difficulties when the simulation cell is small and orbitals interact with their own periodic images; longer simulation cells eliminate these difficulties.

\section{DEFORMED CARBON NANOTUBES}

\section{A. Validation of the technique}

To validate the technique, I begin by investigating singlewalled carbon nanotubes (CNTs). They provide a good benchmark for testing because their mechanical properties were investigated thoroughly in the past [45-50]. For example, it was shown that the energetics of carbon nanotubes with a sufficiently large diameter follow closely the classical thin-sheet elasticity theory with elastic parameters adopted directly from graphene [45,51].

For completeness and for later reference, before proceeding with arbitrary deformations, I verify the thin-sheet model with pure bending, pure stretching, and pure twisting of CNTs. The deformation energies under pure deformations agree very well with pertinent analytical estimates,
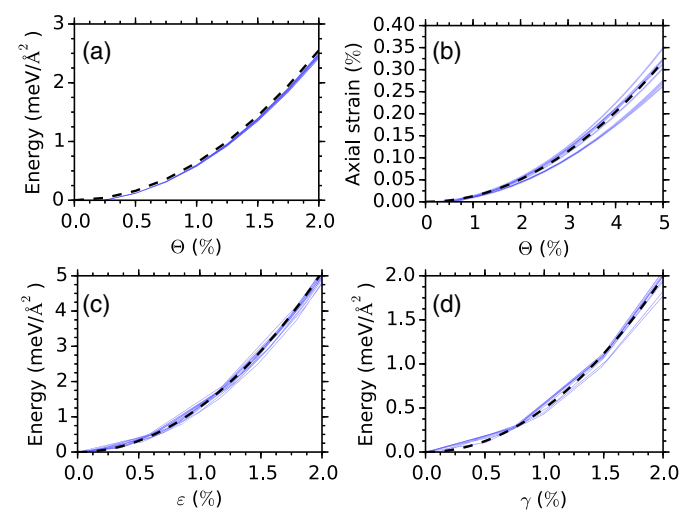

FIG. 2. Validity of thin-sheet elasticity in carbon nanotubes under pure deformations. (The set of selected tubes is listed in Fig. 5.) (a) Energy per sidewall area for CNTs under pure bending. The dashed line is the curve $E / A=\frac{1}{4} Y \Theta^{2}$. (b) Axial stretching induced by pure bending. The dashed line is the curve $\varepsilon_{\Theta}=\frac{3}{4} \beta \Theta^{2}$. (c) Energy per sidewall area for CNTs under pure stretching. Dashed line is the curve $E / A=\frac{1}{2} Y \varepsilon^{2}$. (d) Energy per sidewall area for CNTs under pure twisting. Dashed line is the curve $E / A=\frac{1}{2} G \gamma^{2}$, where $G=Y / 2(1+\sigma)$ is graphene's shear constant.

where the related elastic parameters, the Young's modulus $Y=25.5 \mathrm{eV} / \AA$ and the Poisson ratio $\sigma=0.285$, are calculated for graphene by DFTB (Fig. 2). In addition to linear elasticity, nonlinear (bond anharmonicity) effects cause CNT stretching upon bending, because bonds at inner edge compress less than bonds at outer edge elongate [52]. Assuming a strain-dependent Young's modulus of the form $Y=(1-\beta \varepsilon) Y_{0}$, with the anharmonicity parameter $\beta=1.7$ [52], the analytically calculated axial strain upon bending becomes $\varepsilon_{\Theta}=\frac{3}{4} \beta \Theta^{2}$, as confirmed by simulations [Fig. 2(b)].

To create arbitrary deformations, I use a four-stage deformation path,

$$
(\Theta, \gamma, \varepsilon)(s)=\begin{array}{ll}
\left(\epsilon_{0} s, 0,0\right) & \text { for } s \in[0,1], \\
{\left[\epsilon_{0}, \epsilon_{0}(s-1), 0\right]} & \text { for } s \in[1,2], \\
{\left[\epsilon_{0}, \epsilon_{0}, \epsilon_{0}(s-2)\right]} & \text { for } s \in[2,3], \\
\left(\epsilon_{0}, \epsilon_{0}, \epsilon_{0}\right)(4-s) & \text { for } s \in[3,4]
\end{array}
$$

where $\epsilon_{0}$ is a maximum strain and $s \in[0,4]$ is a deformation coordinate. That is, deforming begins with pure bending, proceeds with additional twisting and then by stretching, and terminates with the synchronous reversal of bending, twisting, and stretching (Video 1). The energy per unit length in a CNT under the deformation $(\Theta, \gamma, \varepsilon)$ is

$$
E(\Theta, \gamma, \varepsilon) / L_{0}=\frac{\pi D Y}{4}\left(\Theta^{2}+\frac{1}{1+\sigma} \gamma^{2}+2 \varepsilon^{2}\right),
$$




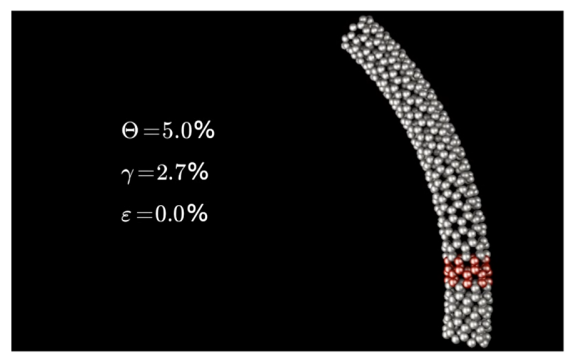

VIDEO 1. Visualization of the deformation path in Eq. (7).

which is a superposition of energies under separate pure deformations (Fig. 2). Now, as demonstrated for an $(11,0)$ CNT, energy from the periodic technique agrees with Eq. (8) to high accuracy [Fig. 3(a)]. That is, deforming a CNT merely by adjusting the symmetry operation $\mathcal{S}_{t}$ gives accurate deformation energies. I emphasize that this agreement is not trivial, because in periodic quantum simulations the elastic properties are a priori indeterminate; they emanate automatically from the electronic structure, from wave functions, and from wave-function symmetries. The agreement can therefore be considered as a direct validation for the technique and a verification of the underlying approximations.
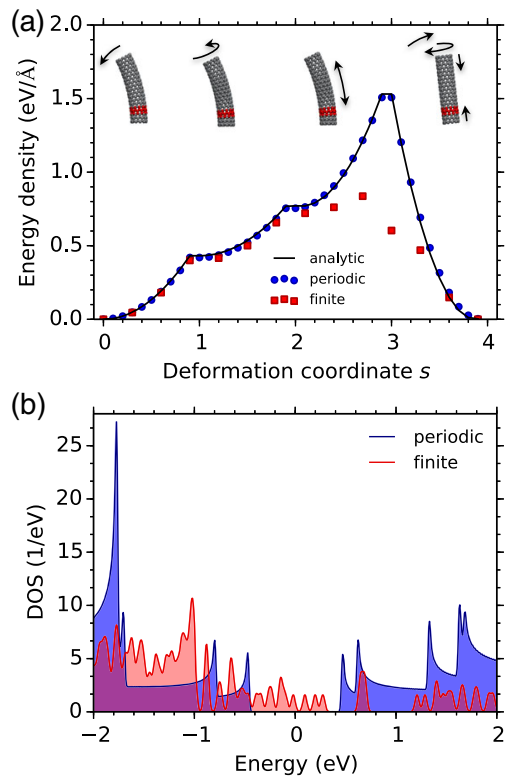

FIG. 3. Comparing periodic and finite calculations. (a) Energy density as a function of the deformation coordinate [the path of Eq. (7)] for an $(11,0)$ CNT using periodic (blue circles) and finite (red squares) simulations. Solid line corresponds to the analytic expression (8); $\epsilon_{0}=5 \%$. (Insets) The periodic simulation cell consists only of the red atoms; the gray atoms are just periodic copies. The CNT of the finite simulation is 25 times the unit cell. (b) Density of states (DOS) of an undeformed $(11,0)$ CNT using periodic (blue; with $200 \kappa$ points) and extended but finite (red; with 1100 atoms) simulations. The Fermi level is at zero.

\section{B. Comparison with finite CNTs}

For comparison, I apply the deformation path (7) also to a finite $(11,0) \mathrm{CNT}$ containing 25 unit cells and 1100 atoms. The deformation is constrained by fixing atoms $I_{\text {bot }}$ near the bottom end and constraining the atoms $I_{\text {top }}$ near the top end to move along a trajectory $\boldsymbol{r}_{I_{\text {top }}}=\mathcal{S}_{t}{ }^{24} \boldsymbol{r}_{I_{\text {bot }}}$. For the finite CNT, Eq. (8) describes the energy well when $s$ is small but poorly when $s$ is large. At around $s \approx 3$, the comparison of energies becomes even more questionable because the end constraints of the finite tube cannot retain the deformation homogeneously and the tube axis loses its circular-arc form [Fig. 3(a)]. In addition, a single energy and force evaluation step takes approximately 1000 times longer for the finite CNT ( $\Delta t \sim 0.5 \mathrm{~h}$ ) than for the periodic CNT ( $\Delta t \sim 2 \mathrm{~s}$ ), and also the number of optimization steps for finite CNT is about 10 times larger.

However, the main difference and the true power of the technique lies in the electronic-structure analysis. Although the atom count in the finite $(11,0) \mathrm{CNT}$ is 1100 , the resulting aspect ratio of 12.4 is trivial compared to the experimental ratios $10^{2-4}$ or even $10^{8}$ [53]. As a result, the density of states (DOS) from finite simulation is unreliable, as it includes spurious end-localized states that arise from quantum finite-size effects [near the Fermi level in Fig. 3(b)]. On the contrary, the electronic structure of a periodic CNT can always be converged by a sufficient number of $\kappa$ points.

\section{Electromechanics under arbitrary deformations}

The DOS for the $(11,0)$ CNT [Fig. 3(b)] is also recorded for the entire deformation path. Upon deforming the van Hove singularities remain prominent, but they shift to higher and lower energies [Fig. 4(a)]. These shifts are reflected by changes of the fundamental energy gap, as reported earlier for pure deformations [52,54-57]. Regarding arbitrary deformations, it turns out that the gap is well described by

$$
\mathcal{E}(\Theta, \gamma, \varepsilon)=\mathcal{E}(0,0,0)+\Delta \mathcal{E}_{\|}\left(\varepsilon_{\Theta}+\varepsilon\right)+\Delta \mathcal{E}_{L}(\gamma),
$$

where $\Delta \mathcal{E}_{\|}(\varepsilon)=\mathcal{E}_{\|}(\varepsilon)-\mathcal{E}_{\|}(0)$ is the gap change under pure axial strain and $\Delta \mathcal{E}_{\angle}(\gamma)$ the corresponding gap change under pure twist [Fig. 4(b)]. Equation (9) also includes contribution from axial strain due to bond anharmonicity, as discussed above [52,57]. This effect is visible at $s<1$, where $\gamma=\varepsilon=0$ but $\varepsilon_{\Theta} \neq 0$ [Fig. 4(b)]. Thus, gap changes under arbitrary deformations are given by linear superposition of gap changes in separate pure deformations. Because the superposition is valid for all van Hove singularities, Eq. (9) is expected to pertain to optical transitions as well [56]. The validity of linear superposition could be anticipated, but it has never been demonstrated directly. 

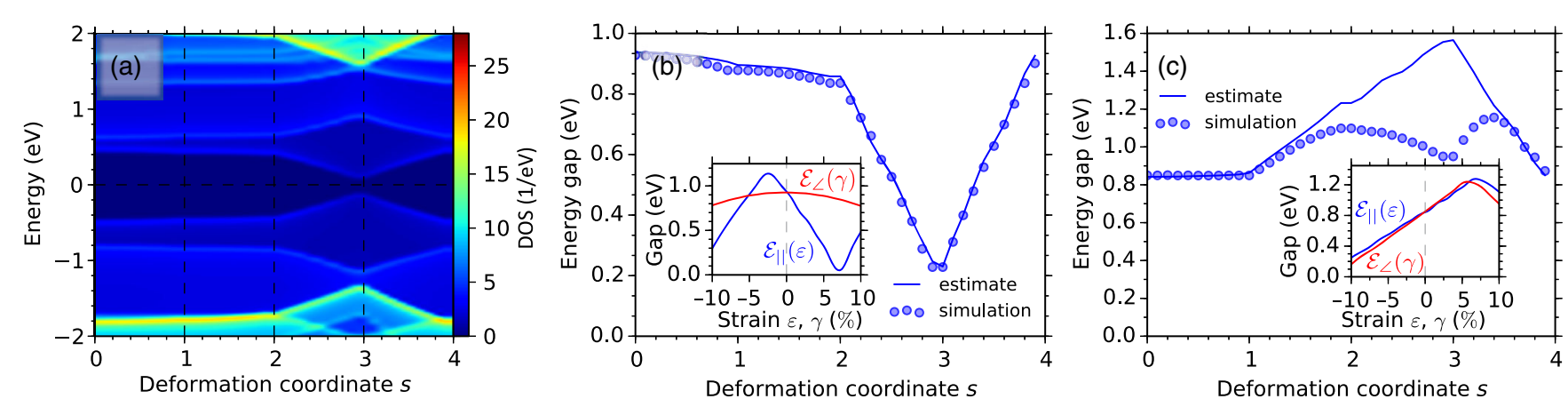

FIG. 4. CNT electromechanics under arbitrary deformations. (a) Contour plot for the density of states (DOS) of (11, 0) CNT under the deformation path of Eq. (7) with $\epsilon_{0}=5 \%$. (b) Energy gap of $(11,0)$ CNT derived from (a) (the circles) and from the estimate of Eq. (9) (the solid line). (Inset) Energy gap of $(11,0) \mathrm{CNT}$ as a function of pure stretching $\left(\mathcal{E}_{\|}\right)$and pure twisting $\left(\mathcal{E}_{\angle}\right)$. (c) The same as $(\mathrm{b})$ for an $(8,4)$ CNT.

The news is, however, that sometimes the superposition principle goes awry without warning. When considering an $(8,4)$ CNT under the path (7) with $\epsilon_{0}=5 \%$, after $s \gtrsim 2$, the gap starts to behave in a manner opposite to that displayed in Eq. (9) [Fig. 2(c)]. In an $(8,4)$ CNT, pure stretching increases the gap [see the inset of Fig. 2(c)], but the stretching of the already-bent-and-twisted tube decreases the gap. The combination of deformations creates a synergy that causes a nonlinear response in the electronic structure and invalidates linear superposition. For an $(8,4) \mathrm{CNT}$, the validity of Eq. (9) is regained by decreasing $\epsilon_{0}$ down to $2 \%$ (not shown), but the limits of validity cannot be anticipated from CNT electromechanics under separate pure deformations; confirmation of possible validity requires explicit simulations with arbitrary deformations.

I repeat the above analysis with $\epsilon_{0}=5 \%$ for a set of different chiral and nonchiral CNTs. It turns out that the superposition equation (9) is most accurate for zigzag and armchair tubes and slightly less accurate for chiral tubes (Fig. 5). The origin for this behavior is unknown and requires further investigation.

\section{Poynting effect}

To return to the mechanical properties of CNTs, previous simulations have already shown that chiral tubes display the Poynting effect, which means that stretching and twisting are coupled (twisting induces stretching, or the other way around) [48]. While predictions have been made using purely classical models, the predictions here are confirmed by a quantum-mechanical method (Fig. 6).

Given the Poynting effect, the bending-induced axial stretching [Fig. 2(b)], and the technique for simulating arbitrary deformations, it is pertinent to investigate whether bending could also induce twisting in CNTs. The twisting angle attributable to bending can be roughly estimated as
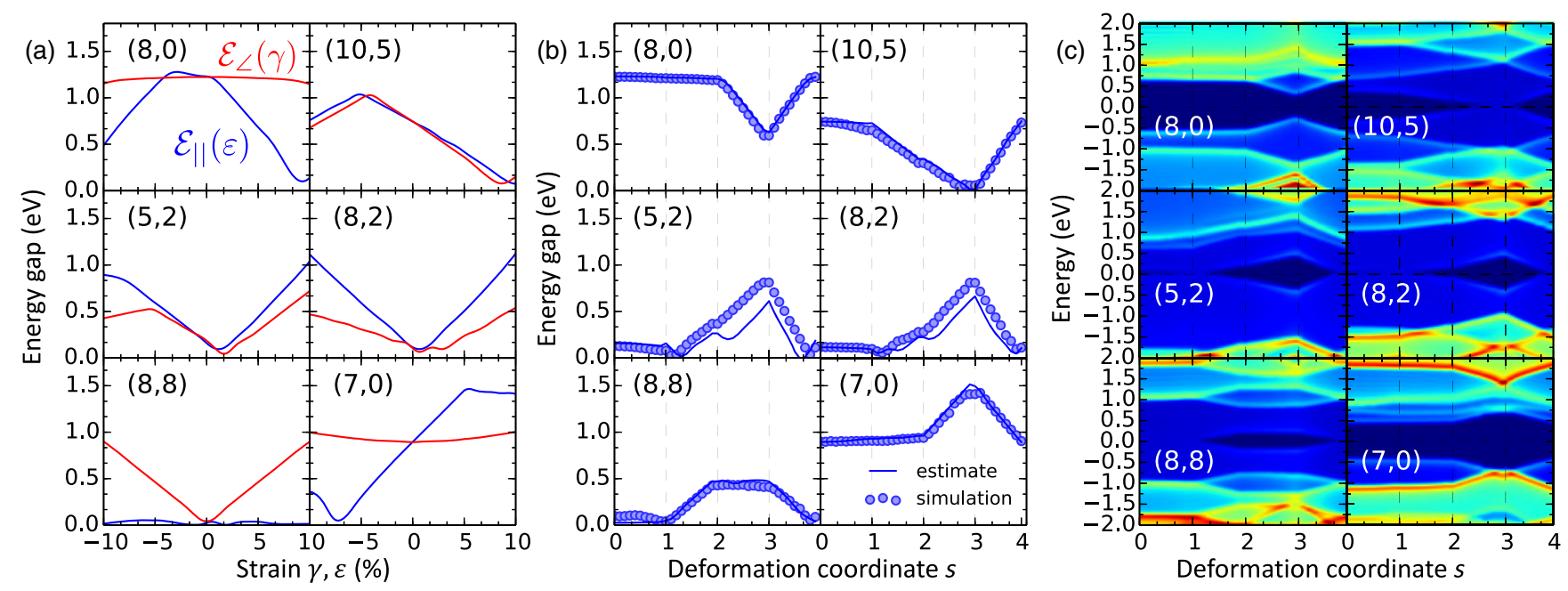

FIG. 5. Electromechanics of various CNTs under arbitrary deformations. (a) Energy gap under pure shear (the red lines) and pure stretch (the blue lines), defining the functions $\mathcal{E}_{\|}(\varepsilon)$ and $\mathcal{E}_{L}(\gamma)$. (b) Energy gaps under the deformation path of Eq. (7). The solid lines are estimates from Eq. (9), using the data from (a). (c) Contour plot for the density of states under the deformation path of Eq. (9). The color scale is the same as in Fig. 4(a). The parameter $\epsilon_{0}=5 \%$ is used in (b) and (c). 


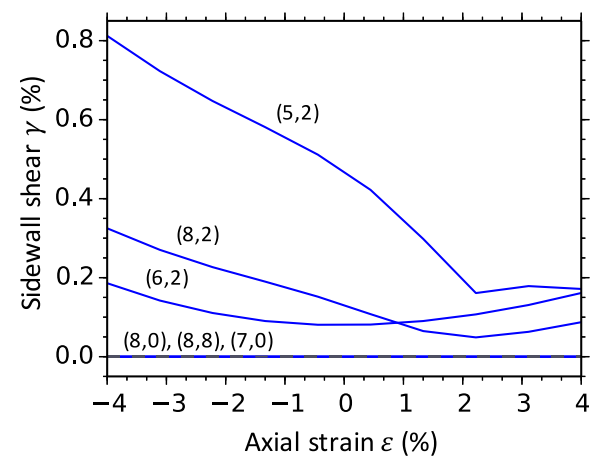

FIG. 6. Poynting effect in CNTs. Panels show the sidewall shear as a function of axial strain. The effect is observed only in chiral tubes, not in nonchiral zigzag $(n, 0)$ or armchair $(n, n)$ tubes.

$\Delta \gamma=|\partial \gamma / \partial \varepsilon| \times \frac{3}{4} \beta \Theta^{2}$, where $|\partial \gamma / \partial \varepsilon|$ measures the magnitude of the Poynting effect. At its maximum, the magnitude is $|\partial \gamma / \partial \varepsilon|_{\max } \approx 0.1$ (Fig. 6), so the largest amount of bending-induced twisting becomes $\Delta \gamma \approx$ $0.13 \times \Theta^{2}$, which turns out to be only $\Delta \gamma \sim 0.1 \%$, even with bending as large as $\Theta=10 \%$. A CNT with $D=1 \mathrm{~nm}$ bent to $\Theta=1 \%$ would then need to be at least a quarter of a millimeter long to twist a full turn. Being this tiny in magnitude, bending-induced twisting could not be resolved in periodic simulations. Zhao and Luo [50] reported twisting-induced bending, but it was due to the Poynting effect combined to a constrained length for a finite tube; intrinsic bending-induced stretching in CNTs seems to be too minor to be of practical significance.

\section{DEFORMED MoS AND Au NANOWIRES}

\section{A. Electromechanics of $\mathrm{Mo}_{6} \mathrm{~S}_{6}$ nanowire}

Let us next leave CNTs aside and move on to study $\mathrm{MoS}_{2}$ monolayer-derived $\mathrm{Mo}_{6} \mathrm{~S}_{6}$ nanowires [42,58-60]. These wires are a timely example of deformed 1D nanostructures, as demonstrated by aberration-corrected transmission electron micrographs [60]. For example, the work of Lin et al. shows highly resilient $\mathrm{Mo}_{6} \mathrm{~S}_{6}$ nanowires bent up to $\Theta \sim 6 \%-10 \%$ [61]. Undeformed $\mathrm{Mo}_{6} \mathrm{~S}_{6}$ wires are metallic, but the twist of magnitude $\gamma=2.7 \%$ (assuming the wire diameter $D=0.3 \mathrm{~nm}$ ) is predicted to open a gap, which is also confirmed here [Fig. 7(a)] [4]. As suggested before, these properties can be exploited in an electromechanical switch that allows current propagation in a straight wire but not in a twisted one [4]. Here, simulations agree with the previous results under pure bending and pure twisting, but the analysis is more transparent, as the band structure can always be plotted for the same minimal cell. Analysis reveals that pure bending creates small energy splittings due to weak wave-function localization at inner and outer sides of the wire [Fig. 7(b)]. Analogous localization has also been reported in the vibrational modes of bent CNTs [37]. Pure bending affects band structure weakly, but a preexisting
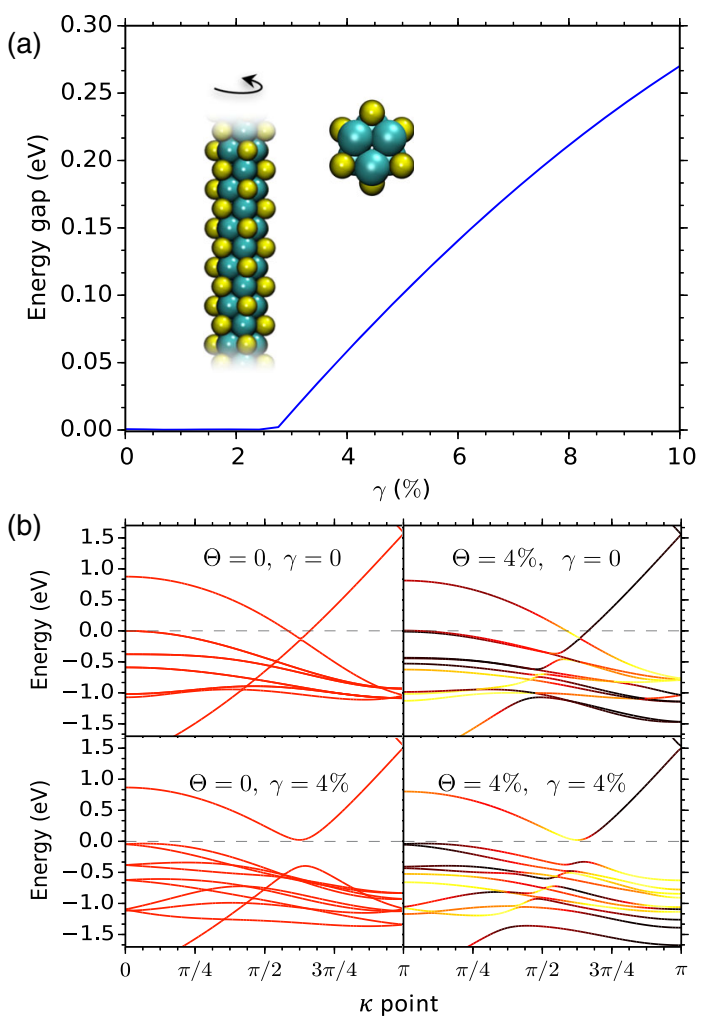

FIG. 7. Electromechanics of $\mathrm{Mo}_{6} \mathrm{~S}_{6}$ nanowire. (a) The energy gap under pure twist (with $D=0.3 \mathrm{~nm}$ ). (Inset) Side and cross-section views of the structure. (b) Band structure under various deformations. The panels correspond to straight (upper left), purely bent (upper right), purely twisted (lower left), and bent and twisted (lower right) wires. For bent wires, the coloring corresponds to wave functions localized more toward inner (darker) and outer (brighter) sides of the wire. The dashed line is the Fermi level.

twist enhances the effect of bending notably [juxtapose the changes in Fig. 7(b) from upper left to upper right with the changes from lower left to lower right]. However, although the twisting-induced metallic-to-semiconducting transition is seen here also under bending, a robust electromechanical switching operation should also require structural robustness; we discuss this matter next.

\section{B. Mechanical stability of $\mathbf{M o}_{6} \mathrm{~S}_{6}$ nanowire}

The reliability of a structural-stability analysis calls for larger simulation cells than the minimal ones. With the minimal 12-atom cell, $\mathrm{Mo}_{6} \mathrm{~S}_{6}$ is stable at least beyond $\Theta=\gamma>4 \%$, as shown above [the lower-right panel in Fig. 7(b)]. When the simulation cell length is extended to $2.6 \mathrm{~nm}$, however, the atomic structure reveals its sensitivity to combined deformations. When twisted, the elastic energy first depends quadratically on $\gamma$, with the torsion constant $150 \mathrm{eV} \AA$, in fair agreement with the literature [59]. Upon further twisting, the energy starts to deflect from this quadratic trend and the wire begins to yield [inset of Fig. 8(a)]. 

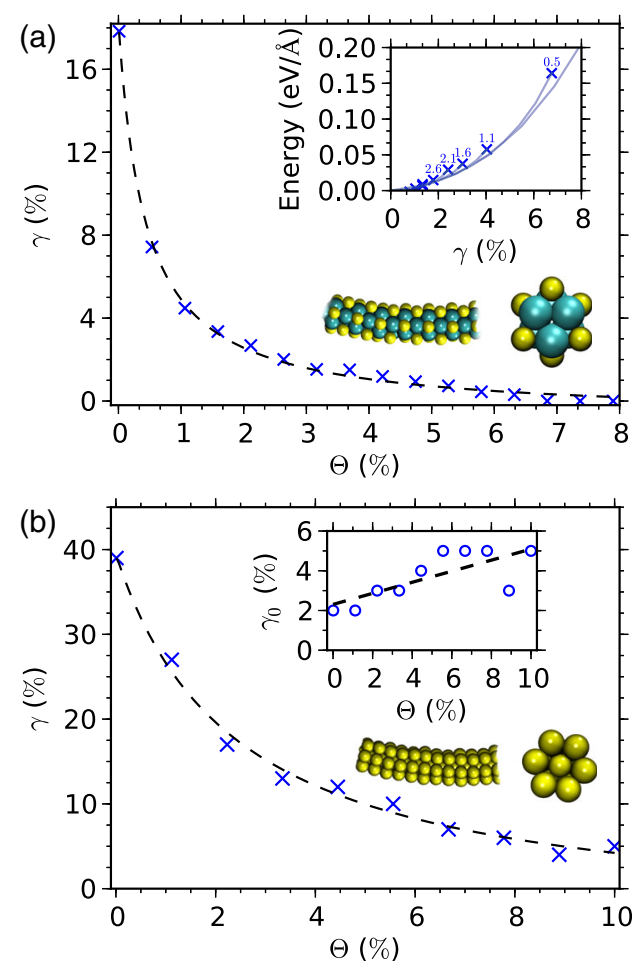

FIG. 8. Yield limits in $\mathrm{Mo}_{6} \mathrm{~S}_{6}$ and $\mathrm{Au}$ nanowires under combined bending and twisting. (a) The twisting yield limit as a function of bending in the $\mathrm{Mo}_{6} \mathrm{~S}_{6}$ nanowire. The dashed line is a fit discussed in the main text. (Inset) Elastic energy as a function of twist for different bends $\Theta$ (numbers shown); yield points are marked with crosses. (b) The twisting yield limit as a function of bending in $\mathrm{Au}_{7}$ nanowire. (Inset) Minimum-energy twists for bent $\mathrm{Au}_{7}$ wires.

Most importantly, the deflection and yielding occurs at a rapidly decreasing twist when bending increases. When plotting the yield points of twisting for different $\Theta$ 's, it becomes evident that combining bending and twisting affects the stability limits dramatically [Fig. 8(a)]. For example, the yield limit in purely twisted wire is $\gamma=18 \%$, but a modest $\Theta \sim 0.5 \%$ bending cuts this limit to less than half. The metalinsulator transition and robust operation of an electromechanical switch device is thus feasible only for relatively straight wires $(\Theta<1.5 \%)$. Besides, temperatures higher than the one used here $(10 \mathrm{~K})$ would probably lower the yield limits even further.

Although a lowering of the twisting yield limit under bending is anticipated, its abruptness is not [Fig. 8(a)]. The yield limit follows the ad hoc form $\left(\gamma+c_{1}\right)\left(\Theta+c_{2}\right)=c_{3}$, where $c_{1}=7.8 \times 10^{-3}, c_{2}=4.2 \times 10^{-3}$, and $c_{3}=8.1 \times 10^{-4}$. This form differs radically from standard yield criteria valid for macroscopic solids, such as the von Mises criterion [62]. The von Mises criterion is based on the fixed allowed energy density and suggests instead a form $b_{1} \gamma^{2}+b_{2} \Theta^{2}=b_{3}$, with constant $b_{i}$ 's. Here, the total energy — and thereby the energy density — at the yield point is not fixed but drops rapidly when $\Theta$ increases [inset of Fig. 8(a)].

\section{Mechanical stability of $\mathrm{Au}_{7}$ nanowire}

I perform a similar stability analysis for a 0.6-nmdiameter $\mathrm{Au}_{7}$ nanowire with 1.7-nm-long cells [43]. This nanowire shows yield limits qualitatively similar to those of $\mathrm{Mo}_{6} \mathrm{~S}_{6}$ [Fig. 8(b)]. Atom trajectories reveal that yielding occurs for the entire wire cross section at once, collectively, which helps us to appreciate the qualitatively different behavior compared to macroscopic rods and wires. Earlier studies of $\mathrm{Mo}_{6} \mathrm{~S}_{6}$ and Au wires revealed several dislocations, which could be indirect indications of the low yield limits under combined deformations [19,59,63]. Yet, this yielding behavior remains a puzzle that deserves further investigation.

As a final observation, bending and twisting in $\mathrm{Au}_{7}$ turn out to be coupled [see the inset of Fig. 8(b)]. At a given $\Theta$, the energy is minimized at the varying twist $\gamma_{0}$, following $\gamma_{0}(\Theta) \approx 0.02+0.28 \times \Theta$. That is, unlike in CNTs, in $\mathrm{Au}_{7}$, bending induces twisting and twisting induces bending, as is familiar from mechanical springs [64].

\section{CONCLUSIONS}

To conclude, I hope to have demonstrated that for a faithful modeling of the mechanical and electromechanical properties of 1D nanostructures, simple modeling with separate pure deformations is insufficient-explicit simulations of combined deformations are mandatory. Demand for such simulations grows as the control over 1D nanostructures improves. Perspective for this demand can be obtained by considering the list of related nanostructure examples, which includes metal, semiconductor and molecular nanowires, DNA, polymers, single- and multiwalled CNTs, CNT ropes and bundles, and nanoribbons of graphene and other 2D materials, among many others. Particularly relevant are their surface functionalizations, which are bound to cause complex deformations.

\section{ACKNOWLEDGMENTS}

I thank Jyri Lahtinen for his contributions during the early stages of the project, Petri Luosma for his comments, the Academy of Finland for the funding (Projects No. 283103 and No. 251216), and the CSC-IT Center for Science in Finland for the computer resources.

[1] R. H. Baughman, A. A. Zakhidov, and W. A. de Heer, Carbon nanotubes-The route toward applications, Science 297, 787 (2002).

[2] Hideaki Ohnishi, Yukihito Kondo, and Kunio Takayanagi, Quantized conductance through individual rows of suspended gold atoms, Nature (London) 395, 780 (1998).

[3] L. M. Jonsson, S. Axelsson, T. Nord, S. Viefers, and J. M. Kinaret, High frequency properties of a CNT-based nanorelay, Nanotechnology 15, 1497 (2004). 
[4] Igor Popov, Sibylle Gemming, Shinya Okano, Nitesh Ranjan, and Gotthard Seifert, Electromechanical switch based on $\mathrm{Mo}_{6} \mathrm{~S}_{6}$ nanowires, Nano Lett. 8, 4093 (2008).

[5] Youmin Rong and Jamie H. Warner, Wired up: Interconnecting two-dimensional materials with one-dimensional atomic chains, ACS Nano 8, 11907 (2014).

[6] Ray H. Baughman, Changxing Cui, Anvar A. Zakhidov, Zafar Iqbal, Joseph N. Barishi, Geoff M. Spinks, Gordon G. Wallace, Alberto Mazzoldi, Danilo De Rossi, Andrew G. Rinzler, Oliver Jaschinski, Siegmar Roth, and Miklos Kertesz, Carbon nanotube actuators, Science 284, 1340 (1999).

[7] M. D. Lima et al., Electrically, chemically, and photonically powered torsional and tensile actuation of hybrid carbon nanotube yarn muscles, Science 338, 928 (2012).

[8] J. Foroughi, G. M. Spinks, G. G. Wallace, J. Oh, M. E. Kozlov, S. Fang, T. Mirfakhrai, J. D. W. Madden, M. K. Shin, S. J. Kim, and R. H. Baughman, Torsional carbon nanotube artificial muscles, Science 334, 494 (2011).

[9] Rufan Zhang, Chong Liu, Po-Chun Hsu, Chaofan Zhang, Nian Liu, Jinsong Zhang, Hye Ryoung Lee, Yingying Lu, Yongcai Qiu, Steven Chu, and Yi Cui, Nanofiber air filters with high-temperature stability for efficient PM2.5 removal from the pollution sources, Nano Lett. 16, 3642 (2016).

[10] P. G. Collins, K. Bradley, M. Ishigami, and A. Zettl, Extreme oxygen sensitivity of electronic properties of carbon nanotubes, Science 287, 1801 (2000).

[11] Yin Cheng, Ranran Wang, Jing Sun, and Lian Gao, A stretchable and highly sensitive graphene-based fiber for sensing tensile strain, bending, and torsion, Adv. Mater. 27, 7365 (2015).

[12] E. T. Thostenson, Z.F. Ren, and T. W. Chou, Advances in the science and technology of carbon nanotubes and their composites: A review, Compos. Sci. Technol. 61, 1899 (2001).

[13] N. Wang, Y. Cai, and R. Q. Zhang, Growth of nanowires, Mater. Sci. Eng. R 60, 1 (2008).

[14] Agnieszka Kuc and Thomas Heine, Shielding nanowires and nanotubes with imogolite: A route to nanocables, Adv. Mater. 21, 4353 (2009).

[15] J.-C. Charlier, A. De Vita, X. Blase, and R. Car, Microscopic growth mechanisms for carbon nanotubes, Science 275, 647 (1997).

[16] X. Tu, S. Manohar, A. Jagota, and M. Zheng, DNA sequence motifs for structure-specific recognition and separation of carbon nanotubes, Nature (London) 460, 250 (2009).

[17] Feng Ding, Avetik R. Harutyunyan, and Boris I. Yakobson, Dislocation theory of chirality-controlled nanotube growth, Proc. Natl. Acad. Sci. U.S.A. 106, 2506 (2009).

[18] Song Jin, Matthew J. Bierman, and Stephen A. Morin, A new twist on nanowire formation: Screw-dislocation-driven growth of nanowires and nanotubes, J. Phys. Chem. Lett. 1, 1472 (2010).

[19] Yi Yu, Fan Cui, Jianwei Sun, and Peidong Yang, Atomic structure of ultrathin gold nanowires, Nano Lett. 16, 3078 (2016).

[20] Hannu Häkkinen, Robert N. Barnett, Andrew G. Schebakov, and Uzi Landman, Nanowire gold chains: Formation mechanisms and conductance, J. Phys. Chem. B 104, 9063 (2000).

[21] J. D. Watson and F. H. Crick, Molecular structure of nucleic acids: A structure for deoxyribose nucleic acid, Nature (London) 171, 737 (1953).

[22] M. Reibold, P. Paufler, A. A. Levin, W. Kochmann, N. Pätzke, and D. C. Meyer, Carbon nanotubes in an ancient Damascus sabre, Nature (London) 444, 286 (2006).

[23] T. Hertel, R. E. Walkup, and P. Avouris, Deformation of carbon nanotubes by surface van der Waals forces, Phys. Rev. B 58, 13870 (1998).

[24] Eilidh Philp, Jeremy Sloan, Angus I. Kirkland, Rüdiger R. Meyer, Steffi Friedrichs, John L. Hutchison, and Malcolm L. H. Green, An encapsulated helical one-dimensional cobalt iodide nanostructure, Nat. Mater. 2, 788 (2003).

[25] D. Golberg, Y. Bando, C. C. Tang, and C. Y. Zhi, Boron nitride nanotubes, Adv. Mater. 19, 2413 (2007).

[26] Ya Qiong Xu, Arthur Barnard, and Paul L. McEuen, Bending and twisting of suspended single-walled carbon nanotubes in solution, Nano Lett. 9, 1609 (2009).

[27] Yihan Zhu, Jiating He, Cheng Shang, Xiaohe Miao, Jianfeng Huang, Zhipan Liu, Hongyu Chen, and Yu Han, Chiral gold nanowires with Boerdijk-Coxeter-Bernal structure, J. Am. Chem. Soc. 136, 12746 (2014).

[28] B. I. Yakobson, C. J. Brabec, and J. Berhnolc, Nanomechanics of Carbon Tubes: Instabilities beyond Linear Response, Phys. Rev. Lett. 76, 2511 (1996).

[29] A. N. Enyashin and A. L. Ivanovskii, The mechanically induced tuning of structural properties for $\mathrm{MgO}$ tubes under uniaxial tension, torsion and bending: Computer molecular modelling, Nanotechnology 18, 205707 (2007).

[30] T. Dumitrica and R. D. James, Objective molecular dynamics, J. Mech. Phys. Solids 55, 2206 (2007).

[31] W. Cai, W. Fong, E. Elsen, and C. R. Weinberger, Torsion and bending periodic boundary conditions for modeling the intrinsic strength of nanowires, J. Mech. Phys. Solids 56, 3242 (2008).

[32] P. Koskinen and O. O. Kit, Efficient Approach for Simulating Distorted Materials, Phys. Rev. Lett. 105, 106401 (2010).

[33] O. O. Kit, L. Pastewka, and P. Koskinen, Revised periodic boundary conditions: Fundamentals, electrostatics and the tight-binding approximation, Phys. Rev. B 84, 155431 (2011).

[34] J. H. Warner, N. P. Young, A. I. Kirkland, and G. A. D. Briggs, Resolving strain in carbon nanotubes at the atomic level, Nat. Mater. 10, 958 (2011).

[35] F. Bloch, Z. Phys. 52, 555 (1929).

[36] J. W. Mintmire, B. I. Dunlap, and C. T. White, Are Fullerene Tubules Metallic?, Phys. Rev. Lett. 68, 631 (1992).

[37] S. Malola, H. Häkkinen, and P. Koskinen, Effect of bending on Raman-active vibration modes of carbonanotubes, Phys. Rev. B 78, 153409 (2008).

[38] O. O. Kit, T. Tallinen, L. Mahadevan, J. Timonen, and P. Koskinen, Twisting graphene nanoribbons into carbon nanotubes, Phys. Rev. B 85, 085428 (2012).

[39] Pekka Koskinen and Oleg O. Kit, Approximate modeling of spherical membranes, Phys. Rev. B 82, 235420 (2010).

[40] D. Porezag, Th. Frauenheim, Th. Köhler, G Seifert, and R. Kaschner, Construction of tight-binding-like potentials on 
the basis of density-functional theory: Application to carbon, Phys. Rev. B 51, 12947 (1995).

[41] P. Koskinen and V. Mäkinen, Density-functional tightbinding for beginners, Comput. Mater. Sci. 47, 237 (2009).

[42] G. Seifert, H. Terrones, M. Terrones, G. Jungnickel, and T. Frauenheim, Structure and Electronic Properties of $\mathrm{MoS}_{2}$ Nanotubes, Phys. Rev. Lett. 85, 146 (2000).

[43] P. Koskinen, H. Häkkinen, G. Seifert, S. Sanna, Th. Frauenheim, and M. Moseler, Density-functional based tight-binding study of small gold clusters, New J. Phys. 8, 9 (2006).

[44] Erik Bitzek, Pekka Koskinen, Franz Gähler, Michael Moseler, and Peter Gumbsch, Structural Relaxation Made Simple, Phys. Rev. Lett. 97, 170201 (2006).

[45] Konstantin Kudin, Gustavo Scuseria, and Boris Yakobson, $\mathrm{C}_{2} \mathrm{~F}, \mathrm{BN}$, and $\mathrm{C}$ nanoshell elasticity from $a b$ initio computations, Phys. Rev. B 64, 235406 (2001).

[46] M. S. Dresselhaus, G. Dresselhaus, J. C. Charlier, and E. Hernández, Electronic, thermal and mechanical properties of carbon nanotubes, Phil. Trans. R. Soc. A 362, 2065 (2004).

[47] C. T. White and J. W. Mintmire, Fundamental properties of single-wall carbon nanotubes, J. Phys. Chem. B 109, 52 (2005).

[48] Haiyi Liang and Moneesh Upmanyu, Axial-Strain-Induced Torsion in Single-Walled Carbon Nanotubes, Phys. Rev. Lett. 96, 165501 (2006).

[49] L. Pastewka, P. Koskinen, C. Elsässer, and M. Moseler, Understanding the microscopic processes that govern the charge-induced deformation of carbon nanotubes, Phys. Rev. B 80, 155428 (2009).

[50] Renjie Zhao and Chenglin Luo, Torsion-induced mechanical couplings of single-walled carbon nanotubes, Appl. Phys. Lett. 99, 231904 (2011).

[51] L. D. Landau and E. M. Lifshitz, Theory of Elasticity, 3rd ed. (Pergamon, New York, 1986).

[52] P. Koskinen, Graphene nanoribbons subject to gentle bends, Phys. Rev. B 85, 205429 (2012).

[53] R. Zhang, Y. Zhang, Q. Zhang, H. Xie, W. Qian, and F. Wei, Growth of half-meter long carbon nanotubes based on Schulz-Flory distribution, ACS Nano 7, 6156 (2013).
[54] C. L. Kane and E. J. Mele, Size, Shape, and Loe Energy Electronic Structure of Carbon Nanotubes, Phys. Rev. Lett. 78, 1932 (1997).

[55] L. Yang and J. Han, Electronic Structure of Deformed Carbon Nanotubes, Phys. Rev. Lett. 85, 154 (2000).

[56] P. Koskinen, Electronic and optical properties of carbon nanotubes under pure bending, Phys. Rev. B 82, 193409 (2010).

[57] P. Koskinen, Electromechanics of twisted graphene nanoribbons, Appl. Phys. Lett. 99, 013105 (2011).

[58] Mahdi Ghorbani-Asl, Nourdine Zibouche, Mohammad Wahiduzzaman, Augusto F. Oliveira, Agnieszka Kuc, and Thomas Heine, Electromechanics in $\mathrm{MoS}_{2}$ and $\mathrm{WS}_{2}$ : Nanotubes vs. monolayers, Sci. Rep. 3, 2961 (2013).

[59] Ai Leen Koh, Shanshan Wang, Can Ataca, Jeffrey C. Grossman, Robert Sinclair, and Jamie H. Warner, Torsional deformations in sub-nanometer MoS interconnecting wires, Nano Lett. 16, 1210 (2016).

[60] Junhao Lin, Yuyang Zhang, Wu Zhou, and Sokrates T. Pantelides, Structural flexibility and alloying in ultrathin transition-metal chalcogenide nanowires, ACS Nano 10, 2782 (2016).

[61] Junhao Lin, Ovidiu Cretu, Wu Zhou, Kazu Suenaga, Dhiraj Prasai, Kirill I. Bolotin, Nguyen Thanh Cuong, Minoru Otani, Susumu Okada, Andrew R. Lupini, Juan-Carlos Idrobo, Dave Caudel, Arnold Burger, Nirmal J. Ghimire, Jiaqiang Yan, David G. Mandrus, Stephen J. Pennycook, and Sokrates T. Pantelides, Flexible metallic nanowires with self-adaptive contacts to semiconducting transition-metal dichalcogenide monolayers, Nat. Nanotechnol. 9, 436 (2014).

[62] R. von Mises, Mechanik der festen Körper im plastisch deformablen Zustand, Math. Phys. Appl. Math. 1, 582 (1913).

[63] Xia Tian, Junzhi Cui, Chaobo Zhang, Zhidong Ma, Rui Wan, and Qi Zhang, Investigations on the deformation mechanisms of single-crystalline $\mathrm{Cu}$ nanowires under bending and torsion, Comput. Mater. Sci. 83, 250 (2014).

[64] S. Klinkel and S. Govindjee, Anisotropic bending-torsion coupling for warping in a non-linear beam, Comput. Mech. 31, 78 (2003). 